\title{
Comparison of the Therapeutic Effects of Rivaroxaban Versus Warfarin in Antiphospholipid Syndrome: A Systematic Review
}

\author{
Mandana KHODASHAHI@, Zahra REZAIEYAZDI@, Maryam SAHEBARI@ \\ Rheumatic Diseases Research Center, Mashhad University of Medical Sciences, Mashhad, Iran
}

\begin{abstract}
Objectives: This study aims to review the studies evaluating the therapeutic effects of rivaroxaban versus those of warfarin in patients with antiphospholipid syndrome (APS).

Materials and methods: The study included randomized clinical trials, comparative studies, cross-sectional investigations, and case series that focused on the effects of warfarin and rivaroxaban and compared the effects of these anticoagulants in patients with APS. The relevant articles published until 2018 were searched in several databases, including PubMed, Scopus, ScienceDirect, Google Scholar, Embase, and Web of Science.

Results: The findings of the reviewed studies showed that rivaroxaban can be used as an effective and safe alternative to warfarin in APS patients. However, the effectiveness of rivaroxaban in the prevention of thrombosis in high-risk APS patients is suspected.

Conclusion: Given the high risk of using rivaroxaban in thrombotic APS patients with labile international normalized ratio (INR) or poor adherence to INR monitoring, it is not suggested to use this agent for these patients.

Keywords: Anticoagulant, antiphospholipid syndrome, rivaroxaban, warfarin.
\end{abstract}

Thrombotic antiphospholipid syndrome (APS) is an autoimmune disorder, which is potentially fatal and devastating., ${ }^{1,2}$ This disorder is the common acquired cause of venous thrombosis, characterized by venous and/or arterial thromboembolism associated with persistent antiphospholipid (aPL) antibodies., ${ }^{3,4}$ Based on a systemic review, $10 \%$ of patients with deep vein thrombosis have aPL antibodies, suggesting the possibility of the underdiagnosis of this syndrome. Thrombotic APS is considered a rare disorder, ${ }^{5}$ which may occur alone or in combination with other autoimmune diseases. APS developed in $15 \%$ of patients with systemic lupus erythematosus (SLE) and is considered a major adverse prognostic factor in these patients. ${ }^{6}$
This syndrome imposes high socioeconomic burden because it can affect every organ. APS is commonly diagnosed based on laboratory findings via the presence of aPL antibodies. ${ }^{7-9}$ Triple positivity (triple-aPL patients) is observed in individuals at high risk of thrombosis. ${ }^{10}$ The management of thrombotic APS is an important measure to minimize its side effects. Oral anticoagulant therapy is a routine approach for the management of venous thromboembolism (VTE) or the reduction of thromboembolic recurrence in patients with thrombotic APS. ${ }^{11-13}$ However, the treatment of thrombotic APS is a challenging issue. The narrow therapeutic window of the medications applied for APS restricts the monitoring of this syndrome. Recurrence

Received: December 27, 2018 Accepted: May 13, 2019 Published online: December 12, 2019

Correspondence: Maryam Sahebari, MD. Rheumatic Diseases Research Center, Mashhad University of Medical Sciences, 9176699199 Mashhad, Iran. Tel: +985138012753 e-mail: sahebarim@mums.ac.ir 
is observed in half of APS patients with an international normalized ratio (INR) below the therapeutic range. Major bleeding is another concern about these patients. ${ }^{14}$

The long-term administration of vitamin $\mathrm{K}$ antagonist (VKA) oral anticoagulants is the main well-known pharmaceutical approach for the management of thrombotic APS. ${ }^{15}$ Warfarin is one of the main VKAs, which is commonly used in the treatment of this syndrome. However, this anticoagulant entails several drawbacks, including the slow onset of action, narrow therapeutic window, as well as drug and alcohol interactions. ${ }^{16}$ Furthermore, the consumption of VKAs by patients with APS is accompanied by some problems. For example, it is difficult to monitor the patients with aPL due to the different reactions of thromboplastin reagents to lupus anticoagulant (LA) and prolonged basal clotting time, which may complicate the diagnosis in patients consuming LA. ${ }^{17}$ The limitations of VKAs underscore the need for searching for new alternative anticoagulants.

A new generation of non-VKA oral anticoagulants has been recently introduced. These anticoagulants are considered as proper options due to their predictable effects, fixed dose, and lack of drug or alcohol interactions. According to the literature, the efficacy and safety of rivaroxaban, dabigatran, apixaban, and edoxaban are similar to those of warfarin in the management of VTE patients. Rivaroxaban (Xarelto) is an oral anticoagulant, which is recognized as an inhibitor of factor Xa. This medication can decrease cerebral bleeding; furthermore, its effect on the prevention of venous and arterial thromboembolism has been proven. ${ }^{18,19}$ With this background in mind, in this study, we aimed to review the studies evaluating the therapeutic effects of rivaroxaban versus those of warfarin in patients with APS.

\section{MATERIALS AND METHODS}

This systematic review was conducted at Mashhad university of Medical sciences between September $1^{\text {st }} 2018$ and November $5^{\text {th }}, 2018$ and performed based on the seven stages recommended in the Cochrane Handbook, including asking a question, selecting the eligibility criteria, searching the literature, excluding and selecting the papers, assessing the quality of the articles, extracting the required information, and presenting data. ${ }^{20}$ This review was conducted on randomized clinical trials (RCTs), comparative studies, cross-sectional investigations, and case series that focused on the effects of rivaroxaban versus those of warfarin in patients with APS and compared the effects of these anticoagulants. The eligible articles published until 2018 were searched in several databases, including PubMed, Scopus, ScienceDirect, Google Scholar, Embase, and Web of Science.

The inclusion criteria were determined based on the participant-intervention-comparisonoutcome-study design. All parallel randomized, quasi-randomized, prospective, and retrospective articles as well as case series including more than three human samples treated with rivaroxaban or both warfarin and rivaroxaban to determine the therapeutic effects of these agents on APS were included.

The exclusion criteria were: (i) publication in languages other than English, (ii) administration of other anticoagulant agents to APS patients, (iii) investigation of the effects of rivaroxaban or both warfarin and rivaroxaban on APS in animal models, and (iv) insufficiency of the presented data or poor description of the applied methods. In addition, nonclinical studies, experimental studies, expert opinions, editorial letters, narrative articles, systematic review, meta-analyses, case reports, consensus statements, and qualitative studies were removed from the review process.

The search process was performed by two specialist researchers, who were continuously in contact with each other to exchange information, via searching PubMed, ScienceDirect, Scopus, Google Scholar, Embase, and Web of Science, from September $1^{\text {st }} 2018$ and November $5^{\text {th }}$, 2018. The search process was accomplished using the following keywords in combination with antiphospholipid syndrome: "Warfarin" or "Coumadin" or "Jantoven" or "Rivaroxaban" or "Xarelto" or "Anticoagulants".

First, the titles and abstracts of all papers were studied, and the articles related to the subject of interest were selected. Then, the abstracts of the related articles were deeply studied. Subsequently, the full texts of the selected papers were obtained, and the articles appearing to meet the inclusion 
criteria were reviewed. The extraction of the information was accomplished using a predesigned data extraction form. The data were independently extracted by the two reviewers after performing an accurate and thorough evaluation.

The search process was independently performed by two researchers and ended on November $5^{\text {th }} 2018$. Finally, after the exclusion of the irrelevant articles, the remaining papers were reviewed meticulously. A standard electronic form was applied to collect the data. The related data, such as the time and place of study, sample size, age of the study population, male/female ratio, bleeding, SLE, medication dosage, duration of treatment, and outcomes, were extracted from all the included papers. Figure 1 depicts the selection process of the papers in a PRISMA flow diagram.

In this study, the papers were assessed based on Cochrane's risk of bias to determine their quality. ${ }^{21}$ Cochrane reviews included two parts containing six specific domains, including sequence generation, allocation concealment, blinding, incomplete outcome data, selective reporting, and other sources of bias. A table was prepared to determine the risk of bias in each of these domains in each article, in which the low and high risks of bias were marked as "Yes" and "No", respectively. Furthermore, an unclear or unknown risk of bias was specified as "Unclear". Figure 1 presents all domains of quality assessment for each study. This process was performed twice by the two independent assessors during the data extraction process. Figure 2 illustrates the level of evidence of included articles.

\section{RESULTS}

The search process resulted in the identification of 918 papers. Studies focused on anticoagulant agents other than warfarin and rivaroxaban in patients with APS $(n=26)$ and those investigating animals $(n=2)$ were removed from the review process. Additionally, one, three, and 15 articles were excluded due to the inaccessibility of the fulltext version, insufficient data, and experimental or in vitro design, respectively. Additionally, the papers in the form of editorial letters $(n=7)$, books $(n=1)$, case reports $(n=29)$, qualitative and narrative articles $(\mathrm{n}=24)$, and systematic reviews $(n=3)$ were removed from the study.

Finally, 14 articles $^{1,22-34}$ were included in the study after the detailed evaluation of the full-text versions. Table 1 tabulates some of the variables examined in the reviewed studies, including the type of study, age of the participants, type of

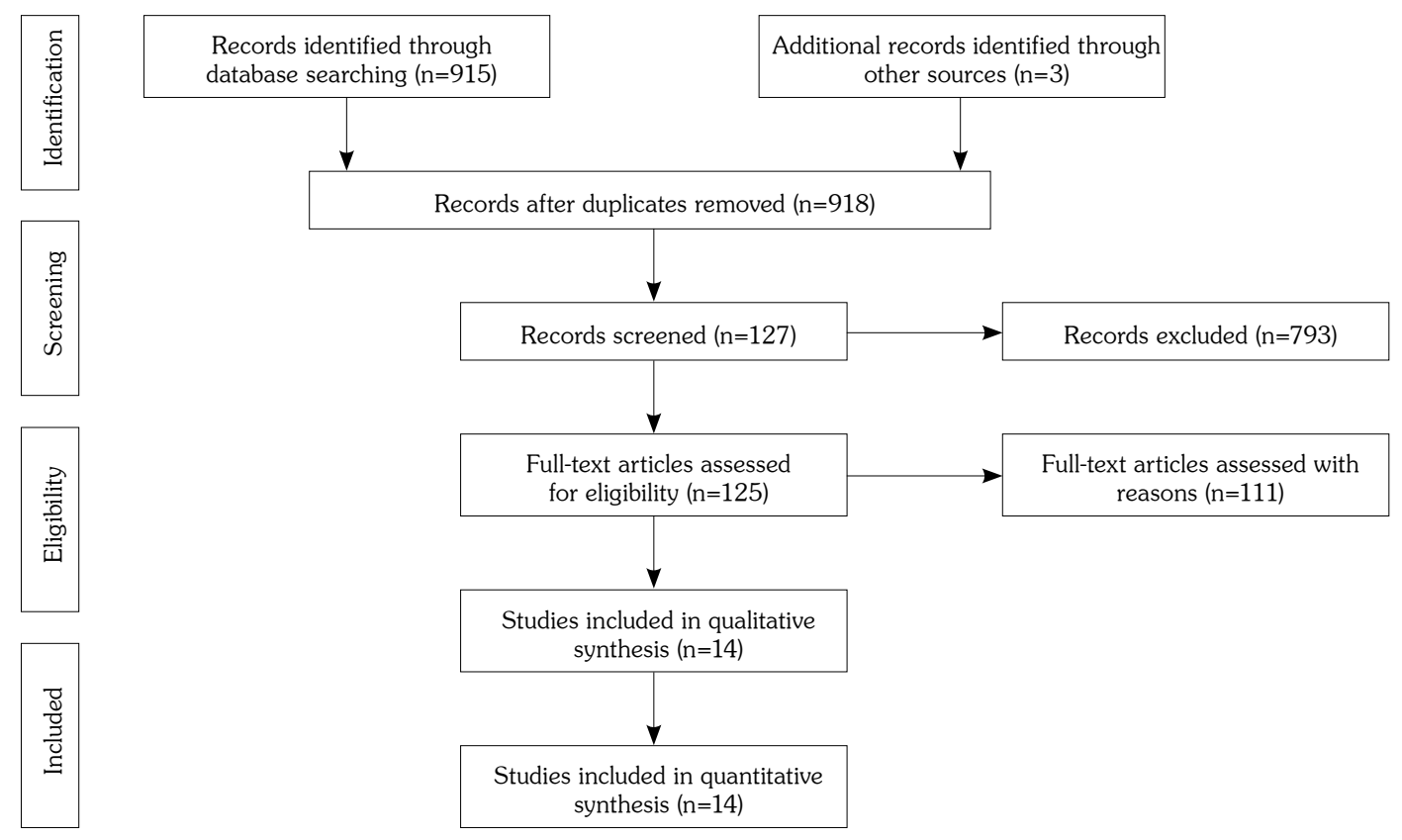

Figure 1. PRISMA flow diagram showing selection process of papers. 


\begin{tabular}{|c|c|c|c|c|c|c|c|c|c|c|c|c|}
\hline & 吾 & 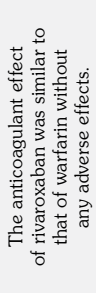 & 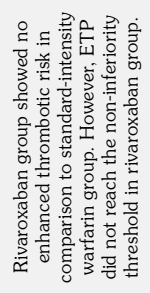 & 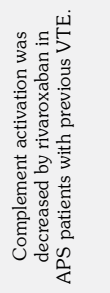 & 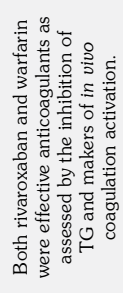 & 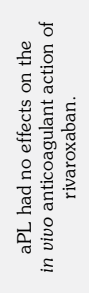 & 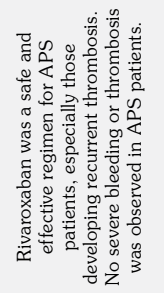 & 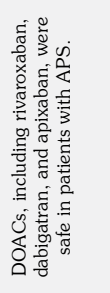 & 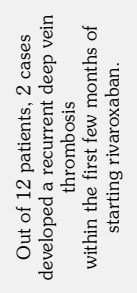 & 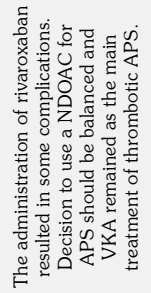 & 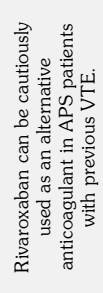 & 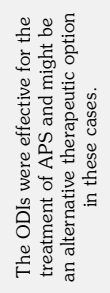 \\
\hline & & 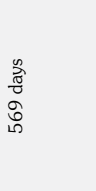 & 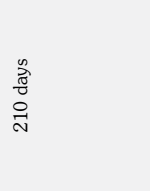 & $\stackrel{2}{z}$ & $\stackrel{8}{z}$ & z̊ & 品 & $\approx$ & $\stackrel{\circ}{z}$ & 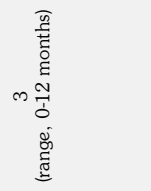 & 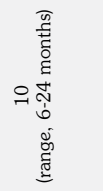 & 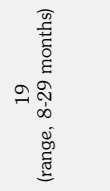 \\
\hline & 焉 & & 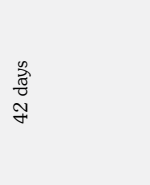 & 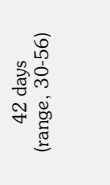 & 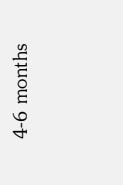 & 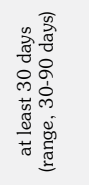 & 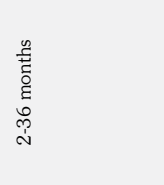 & 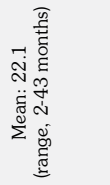 & 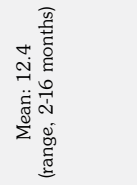 & 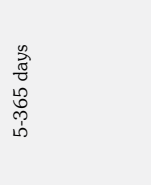 & ' & \\
\hline & 畹 & 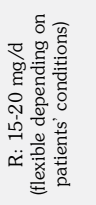 & 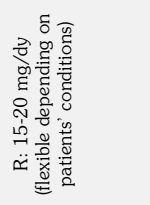 & $\begin{array}{l}\frac{3}{5} \\
\text { oो } \\
\frac{1}{N}\end{array}$ & 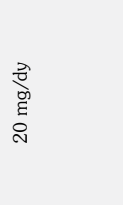 & 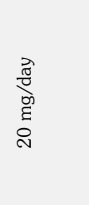 & 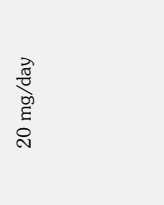 & 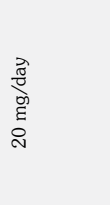 & 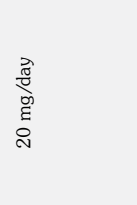 & 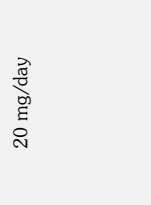 & 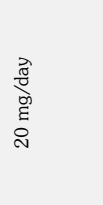 & 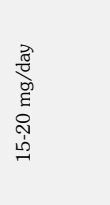 \\
\hline & 惢 & $\vec{\sigma}$ & $\stackrel{\partial}{ }$ & $\stackrel{\partial}{ }$ & , & 胥 & ' & 8 & $\stackrel{m}{\infty}$ & & ' & \\
\hline & 营山 & 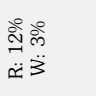 & 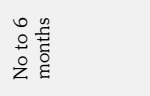 & & . & zo & 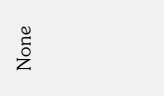 & $\begin{array}{l}\text { ơ } \\
\text { dु } \\
\text { b }\end{array}$ & 总 & 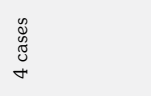 & 气ั̆ & ֻू. \\
\hline & 莺 & 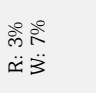 & z̊ & , & z̆ & ż & 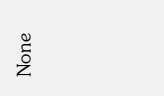 & 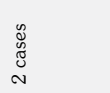 & & & 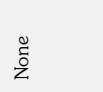 & 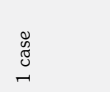 \\
\hline 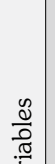 & $\stackrel{\Upsilon}{\underline{z}}$ & 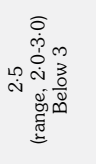 & 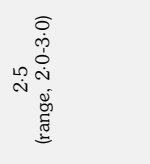 & & 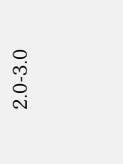 & 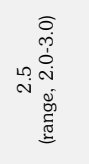 & 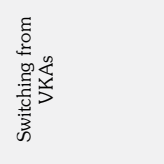 & 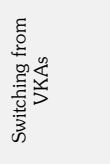 & 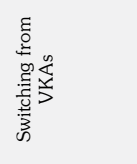 & 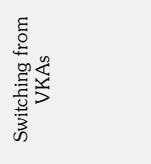 & 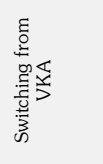 & 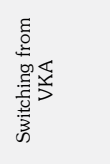 \\
\hline 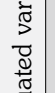 & 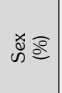 & 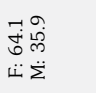 & 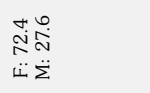 & 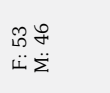 & 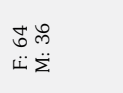 & & & $\begin{array}{l}\text { જت } \\
\ddot{\sim} \ddot{\Sigma}\end{array}$ & 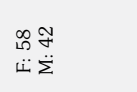 & 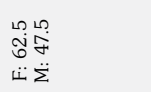 & 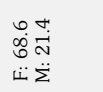 & 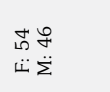 \\
\hline 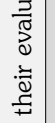 & 赵 & 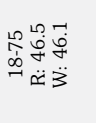 & 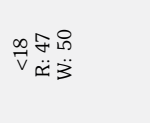 & f & 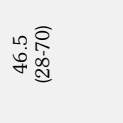 & & & 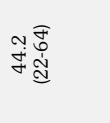 & 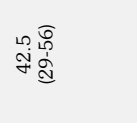 & 蛋 & 㭲 & 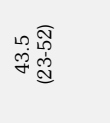 \\
\hline $\begin{array}{l}\bar{z} \\
\bar{c} \\
0 \\
0 \\
\bar{z} \\
\bar{z}\end{array}$ & 产: & 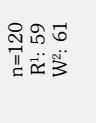 & 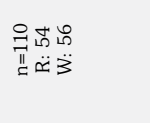 & 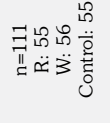 & 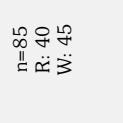 & 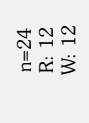 & 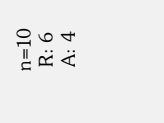 & 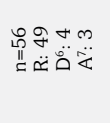 & $\stackrel{N}{\mathbb{I}}$ & $\stackrel{\text { II }}{=}$ & $\stackrel{\mathscr{n}}{\mathbb{1}}$ & $\begin{array}{l}\stackrel{2}{2}=7 \\
\ddot{\circ} \ddot{0}\end{array}$ \\
\hline 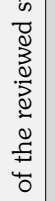 & 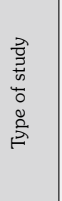 & 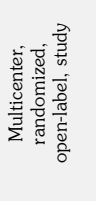 & 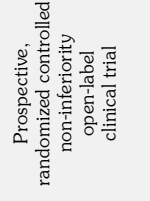 & 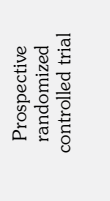 & 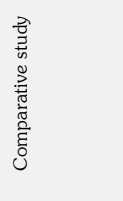 & 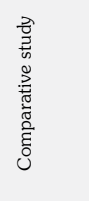 & 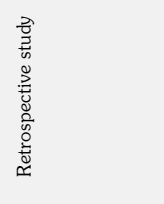 & 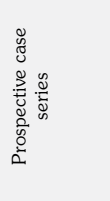 & 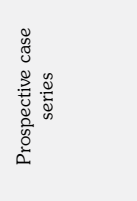 & 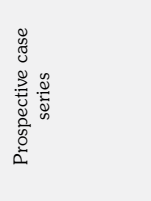 & 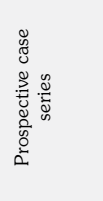 & 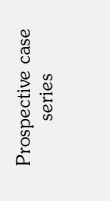 \\
\hline 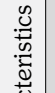 & $\begin{array}{l}\text { 吾 } \\
\text { 言 }\end{array}$ & 胥 & 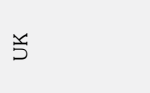 & $\check{\check{J}}$ & 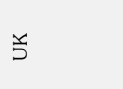 & 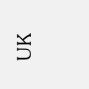 & 崩 & 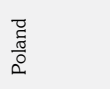 & 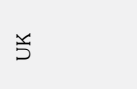 & 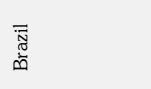 & $\frac{2}{\underline{\underline{E}}}$ & 总 \\
\hline 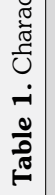 & 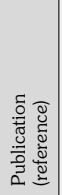 & 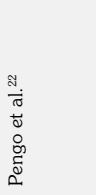 & 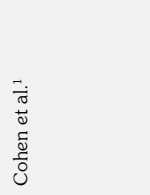 & 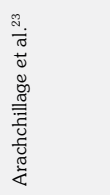 & 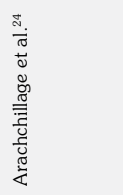 & 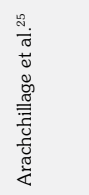 & 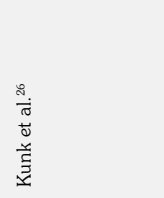 & 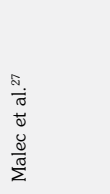 & 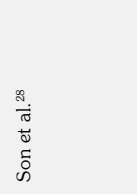 & 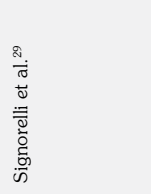 & 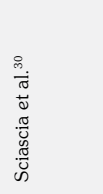 & 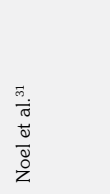 \\
\hline
\end{tabular}




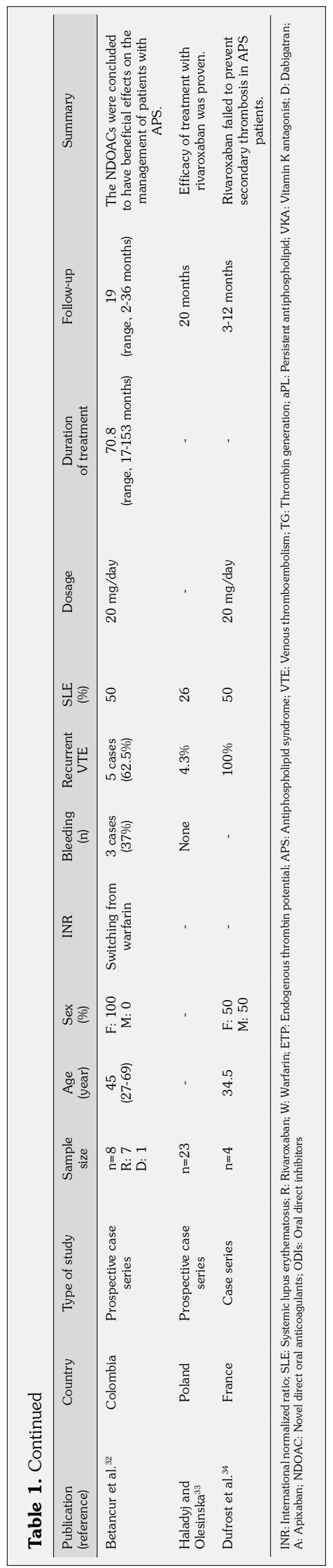

study, sample size, age of the study population, male/female ratio, bleeding, incidence of SLE, medication dosage, treatment duration, follow-up, and outcomes. Given the impossibility of the

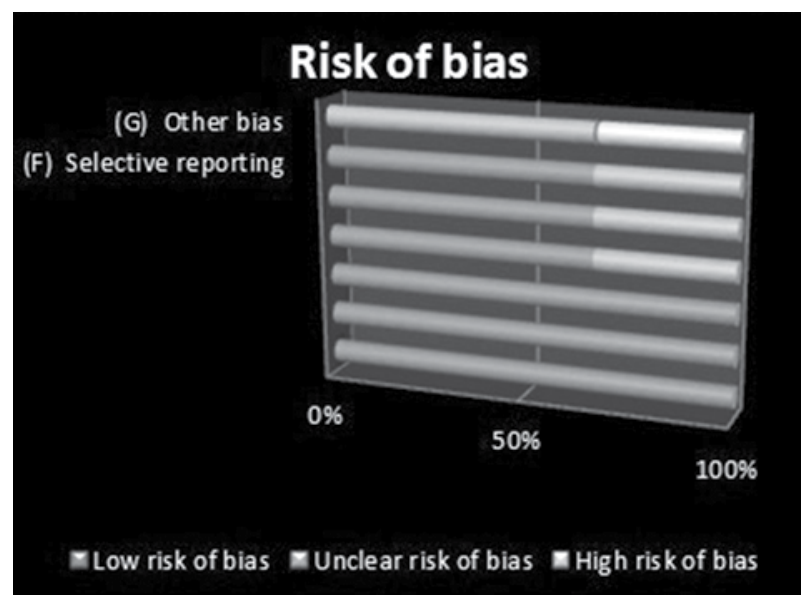

\begin{tabular}{|l|c|c|c|c|c|c|c|}
\hline $\begin{array}{l}\text { Publication } \\
\text { (reference) }\end{array}$ & A & B & C & D & E & F & G \\
\hline Pengo et al. ${ }^{22}$ & + & + & + & + & + & + & ?? \\
\hline Cohen et al. $^{1}$ & + & + & + & + & $? ?$ & + & - \\
\hline Arachchillage et al. $^{23}$ & + & + & + & ?? & + & ?? & ?? \\
\hline
\end{tabular}

Figure 2. Quality assessment of five randomized control trials included in review process in terms of level of evidence.

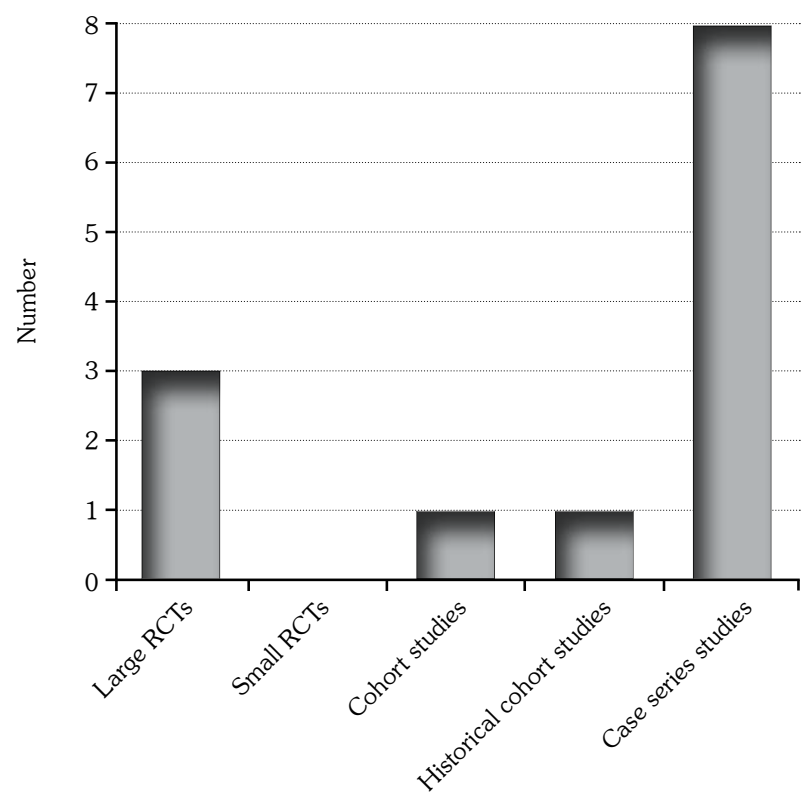

Figure 3. Assessment of the articles included in tem of levels of evidence.

RCTs: Randomized clinical trials. 
quality assessment of the studies other than RCTs; as depicted in Figure 2, a diagram was designed to evaluate all included articles in terms of the level of evidence. ${ }^{35}$ The included studies were performed in 13 different countries. In this respect, 11, one, and two articles were conducted in Europe, North America, and South America, respectively. In terms of the sample size, three (20\%) articles adopted a large study population all of which were RCTs. Moreover, the sample size of two (13.3\%) articles was moderate, and it was small in nine (47.36\%) papers, eight of which were case series. These studies were performed on 921 patients, the majority of whom were female. The patients had an age range of 34-54 years. The INR ranged within $2-4$, and rivaroxaban was daily administered at a dosage range of $15-20 \mathrm{mg}$. The rates of bleeding in patients treated with rivaroxaban and warfarin were obtained as $0-27 \%$ and $0-3.1 \%$, respectively.

Recurrent VTE was not observed in three studies after treatment with rivaroxaban $(n=2)$ or warfarin $(n=1)$. Furthermore, the patients treated with warfarin had a lower rate of recurrent VTE, compared to those administered rivaroxaban. The frequency of SLE was estimated at 16-60\% in APS patients. The duration of treatment varied across different articles (i.e., from two days to several months), and the majority of the papers involved follow-ups. Regarding the outcome, in most of the studies, rivaroxaban and warfarin were reported to have positive effects on the treatment course of APS patients.

\section{DISCUSSION}

Warfarin is the most common type of VKA, which is considered as the current treatment of thrombotic APS patients. ${ }^{36,37}$ The use of VKA is accompanied by some problems, such as drug and food interactions and teratogenicity. On the other hand, patients managed with warfarin need to receive frequent INR monitoring and may respond to treatment differently. ${ }^{38,39}$ It seems that the novel direct oral anticoagulant (NDOAC), including direct thrombin inhibitor (e.g., dabigatran) and direct anti-Xa inhibitors (e.g., rivaroxaban, apixaban, and edoxaban), can play an effective role in the prevention of thrombosis in APS patients through the direct inhibition of a single enzyme of the coagulation cascade. ${ }^{40}$ The other advantages of NDOAC include the lack of need for laboratory monitoring and low drug and food interactions. However, given the lack of antidotes, an increased risk of major bleeding has remained as the main limitation of these agents $\left(0.57-10 \%\right.$ per year). ${ }^{41}$

Although rivaroxaban and warfarin have a different mechanism for the inhibition of thrombin generation, the effectiveness of both agents in the inhibition of thrombin generation in patients with VTE has been confirmed. ${ }^{24,42}$ Cohen et al. ${ }^{1}$ could not observe the non-inferiority threshold in endogenous thrombin potential (ETP) after using rivaroxaban. However, they concluded that rivaroxaban could be used as an effective and safe alternative treatment in APS patients due to observing no difference between rivaroxaban and warfarin groups in terms of thrombotic risk in peak thrombin generation. In the mentioned study, few reported cases with raised coagulation activation marker concentrations were reported without any thrombotic events or major bleeding during the six-month follow-up. With regard to the high quality of life in APS patients treated with rivaroxaban in comparison to that in patients receiving warfarin, the use of this drug was suggested in the mentioned study.

The functional levels of vitamin-K-dependent coagulation factors are reduced by warfarin; furthermore, this agent is reported to equally affect all phases of thrombin generation. On the other hand, rivaroxaban acts via the direct inhibition of factor Xa by binding to its active site. Formation of the prothrombinase complex due to the initiation and diffusion of thrombin generation occurs after using rivaroxaban. ${ }^{43,44}$ This leads to the protraction of thrombin generation curve and prolongation of the lag time and the time to peak thrombin generation, thereby increasing ETP more than the extent considered for the degree of anticoagulation. ${ }^{45}$ Cohen et al. ${ }^{1}$ showed an increase in ETP and time to peak thrombin generation in the majority of patients after changing the agent from warfarin to rivaroxaban. Moreover, the peak thrombin generation was decreased by $40 \%$, indicating the anticoagulation characteristics of rivaroxaban.

There is a possible interaction between aPL antibodies and anticoagulant action of direct oral anticoagulants; however, no interaction 
occurs between the effects of rivaroxaban and LA positivity on thrombin generation. ${ }^{24}$ The clinical efficacy and long-term safety of rivaroxaban in the treatment of patients with thrombotic APS have not been confirmed yet. Some studies have evaluated the mechanism of the action of this agent in the APS patients; ${ }^{1,24}$ however, they have some limitations. Regarding this, comprehensive studies are required with large sample sizes, low bias risks, and longer follow-ups considering all aspects to obtain a definitive result.

It should be noted that thrombotic APS is clinically heterogeneous. Therefore, given the probability of the recurrence of this problem, the determination of the exact dosage of anticoagulant is a matter of fundamental importance, which depends on the clinical phenotype. ${ }^{46}$ According to Arachchillage et al. ${ }^{24}$ the reduction induced by rivaroxaban in complement activation may provide an additional benefit to the anticoagulant effect of this agent. The interference between aPL antibodies and anticoagulant may be observed between oral anticoagulants. Due to the sensitivity of thromboplastins to LAs, the monitoring of patients with APS is a matter of paramount significance because the anticoagulant intensity may not be reflected in INR. When developing INR instability, it is required to perform frequent anticoagulant monitoring with undetermined time intervals. ${ }^{47}$ One of the advantages of DOACs over warfarin is their lack of need for routine anticoagulation monitoring.

The anticoagulant effect of rivaroxaban in APS patients depends on the treatment regimen; accordingly, there is no specified concentration range for the clinical application of this agent. Rivaroxaban leads to peak-to-trough fluctuations during the treatment, while the treatment effect of warfarin is constant. Based on population pharmacokinetics, peak rivaroxaban concentrations range within 160-360 $\mu \mathrm{g} / \mathrm{L} .{ }^{48}$ The lack of APS recurrence during a six-month follow-up in patients using rivaroxaban confirmed the switching of anticoagulants from warfarin to oral rivaroxaban. Triple positivity for LA and antibodies against cardiolipin and beta2glycoprotein I at baseline was observed in nearly one-third of the patients treated with rivaroxaban. ${ }^{10,48}$
It seems that the administration of DOAC is a safer approach with a severe bleeding rate of $3.6 \%$ per year in comparison with VKA treatment with a bleeding risk of up to $10 \% .{ }^{49}$ Based on a study performed by Patel et al., ${ }^{50}$ the patients receiving rivaroxaban had a lower rate of intracranial and fatal bleeding, compared to those using warfarin; however, there was no significant difference between them in terms of the risk of major bleeding. On the other hand, Cohen et al. ${ }^{51}$ showed no thrombosis or major bleeding after 210 days of treatment with rivaroxaban or warfarin. Furthermore, in another study carried out by Kunk et al. ${ }^{20}$ on 10 APS patients treated with DOACs, no severe bleeding or thrombosis was reported during a three-year follow-up. These findings support the effectiveness of rivaroxaban and other DOACs in APS patients. However, Win and Rodgers ${ }^{52}$ reported the recurrence of thrombosis in three severe APS cases treated with rivaroxaban and dabigatran.

In a study performed by Cervera et al., ${ }^{49}$ the rate of recurrent thrombosis in APS patients treated with anticoagulants was 25\% within a 10 -year period. The annual rate of recurrent thrombosis in APS patients treated with warfarin was estimated at $1.5 \% .^{14,53}$ Accordingly, the therapeutic effect of DOACs in these patients is similar to that of warfarin. In a study, the overall thrombotic risk was not increased in the APS patients treated with rivaroxaban, compared to that in the patients managed with warfarin, which was confirmed by the lack of new thrombosis or major bleeding and a low rate of clinically relevant bleeding. ${ }^{48}$ Therefore, future studies are recommended to investigate the efficacy of DOACs in APS patients without recurrent VTE or those with stroke or other arterial thrombosis.

Anticoagulant therapy is a necessary approach for the management of patients with APS. However, in some cases, long-term VKA administration necessitates the frequent control of INR, thereby posing problems in the treatment process. Moreover, drug and dietary interaction is a basic problem in APS patients. There is a risk of thrombosis recurrence in APS patients treated with VKA due to its long half-life and INR lability; however, it is a rare condition. These limitations are less 
frequently observed in DOACs. ${ }^{54}$ The possibility of predicting the pharmacokinetic effects of DOAC therapy eliminates the need for diet adjustments and routine laboratory monitoring. ${ }^{1}$ Although DOACs entail some advantages, their short half-life may result in a high recurrence risk. Moreover, it is not possible to ensure complete treatment in the cases undergoing DOAC therapy. Therefore, switching from VKA to DOACs is not appropriate in patients with poor adherence to VKA.

The current review has some limitations. The majority of the studies evaluating DOAC efficacy in APS patients were case series and case reports; therefore, we had no sufficient data in this regard. There are limited studies on the effectiveness of warfarin and rivaroxaban in APS patients; moreover, the majority of the publications in this regard have high bias and low evidence level; therefore, we cannot obtain certain results. Regarding this, the results of our research should be used cautiously. There are few randomized controlled trials investigating the efficacy and safety of warfarin and rivaroxaban in APS patients. It is recommended to further examine the effectiveness of DOACs in APS patients while considering clinical endpoints, such as thrombosis, bleeding, stork, and death, using longer follow-ups.

In conclusion, the effectiveness of rivaroxaban in the prevention of thrombosis in high-risk APS patients is suspected. This should be confirmed by future randomized controlled trials focused on clinical endpoints. Given the high risk of using rivaroxaban in thrombotic APS patients with labile INR or poor adherence to INR monitoring, this agent is not suggested for such patients.

\section{Acknowledgments}

We thank Ms. Massomeh Golmohammadi for her assistance in the preparation of this article.

\section{Declaration of conflicting interests}

The authors declared no conflicts of interest with respect to the authorship and/or publication of this article.

\section{Funding}

The authors received no financial support for the research and/or authorship of this article.

\section{REFERENCES}

1. Cohen $\mathrm{H}$, Hunt BJ, Efthymiou M, Arachchillage DR, Mackie IJ, Clawson S, et al. Rivaroxaban versus warfarin to treat patients with thrombotic antiphospholipid syndrome, with or without systemic lupus erythematosus (RAPS): a randomised, controlled, open-label, phase $2 / 3$, non-inferiority trial. Lancet Haematol 2016;3:e426-36.

2. Ahluwalia J, Sreedharanunni S, Kumar N, Masih J, Bose SK, Varma N, et al. Thrombotic Primary Antiphospholipid Syndrome: the profile of antibody positivity in patients from North India. Int $J$ Rheum Dis 2016;19:903-12.

3. de Groot PG, de Laat B, Rand J, Vlachoyiannopoulos PG, El-Assaad F, Krilis SA, et al. Natural Proteins Involved in Antiphospholipid Syndrome. In: Erkan D, Lockshin MD, editors. Antiphospholipid syndrome. Cham: Springer; 2017. p. 15-27.

4. Pengo V, Denas G. Diagnostics and treatment of thrombotic antiphospholipid syndrome (APS): A personal perspective. Thromb Res 2018;169:35-40.

5. Andreoli L, Chighizola CB, Banzato A, Pons-Estel GJ, Ramire de Jesus G, Erkan D. Estimated frequency of antiphospholipid antibodies in patients with pregnancy morbidity, stroke, myocardial infarction, and deep vein thrombosis: a critical review of the literature. Arthritis Care Res (Hoboken) 2013;65:1869-73.

6. Ruiz-Irastorza G, Egurbide MV, Ugalde J, Aguirre C. High impact of antiphospholipid syndrome on irreversible organ damage and survival of patients with systemic lupus erythematosus. Arch Intern Med 2004;164:77-82.

7. Pierangeli SS, de Groot PG, Dlott J, Favaloro E, Harris EN, Lakos G, et al. 'Criteria' aPL tests: report of a task force and preconference workshop at the 13th International Congress on Antiphospholipid Antibodies, Galveston, Texas, April 2010. Lupus 2011;20:182-90.

8. Keswani SC, Chauhan N. Antiphospholipid syndrome. J R Soc Med 2002;95:336-42.

9. Forastiero R. Multiple antiphospholipid antibodies positivity and antiphospholipid syndrome criteria re-evaluation. Lupus 2014;23:1252-4.

10. Pengo V, Ruffatti A, Legnani C, Gresele P, Barcellona $\mathrm{D}$, Erba N, et al. Clinical course of high-risk patients diagnosed with antiphospholipid syndrome. J Thromb Haemost 2010;8:237-42.

11. Keeling D, Baglin T, Tait C, Watson H, Perry D, Baglin $\mathrm{C}$, et al. Guidelines on oral anticoagulation with warfarin - fourth edition. $\mathrm{Br} \mathrm{J}$ Haematol 2011;154:311-24.

12. Pengo V, Ruffatti A, Tonello M, Cuffaro S, Banzato A, Bison E, et al. Antiphospholipid syndrome: antibodies to Domain 1 of $\beta 2$-glycoprotein 1 correctly classify patients at risk. J Thromb Haemost 2015;13:782-7.

13. Bungard TJ, Semchuk W. The management of venous thromboembolism: A practical tool for the front-line clinician. Can Pharm J (Ott) 2017;150:81-9. 
14. Crowther MA, Ginsberg JS, Julian J, Denburg J, Hirsh J, Douketis J, et al. A comparison of two intensities of warfarin for the prevention of recurrent thrombosis in patients with the antiphospholipid antibody syndrome. N Engl J Med 2003;349:1133-8.

15. Zirlik A, Bode $\mathrm{C}$. Vitamin $\mathrm{K}$ antagonists: relative strengths and weaknesses vs. direct oral anticoagulants for stroke prevention in patients with atrial fibrillation. J Thromb Thrombolysis 2017;43:365-79.

16. Cohen H, Doré CJ, Clawson S, Hunt BJ, Isenberg $\mathrm{D}$, Khamashta $\mathrm{M}$, et al. Rivaroxaban in antiphospholipid syndrome (RAPS) protocol: a prospective, randomized controlled phase II/ III clinical trial of rivaroxaban versus warfarin in patients with thrombotic antiphospholipid syndrome, with or without SLE. Lupus 2015;24:1087-94.

17. Pengo V, Tripodi A, Reber G, Rand JH, Ortel TL, Galli $\mathrm{M}$, et al. Update of the guidelines for lupus anticoagulant detection. Subcommittee on Lupus Anticoagulant/Antiphospholipid Antibody of the Scientific and Standardisation Committee of the International Society on Thrombosis and Haemostasis. J Thromb Haemost 2009;7:1737-40.

18. Bauersachs R, Berkowitz SD, Brenner B, Buller HR, Decousus H, Gallus AS, et al. Oral rivaroxaban for symptomatic venous thromboembolism. N Engl J Med 2010;363:2499-510.

19. Mega JL, Braunwald E, Wiviott SD, Bassand JP, Bhatt DL, Bode $\mathrm{C}$, et al. Rivaroxaban in patients with a recent acute coronary syndrome. $\mathrm{N}$ Engl $\mathrm{J}$ Med 2012;366:9-19.

20. Green S, Higgins J. Cochrane handbook for systematic reviews of interventions. Version; 2005. Available at: http://www.mri.gov.lk/assets/Uploads/Research/ Cochrane-Hand-booktext.pdf

21. Higgins J. Cochrane handbook for systematic reviews of interventions. Version 5.1.0. The Cochrane Collaboration. Available at: http://handbook-5-1. cochrane.org [Updated: March 2011].

22. Pengo V, Denas G, Zoppellaro G, Jose SP, Hoxha A, Ruffatti A, et al. Rivaroxaban vs warfarin in highrisk patients with antiphospholipid syndrome. Blood 2018;132:1365-71.

23. Arachchillage DR, Mackie IJ, Efthymiou M, Chitolie A, Hunt BJ, Isenberg DA, et al. Rivaroxaban limits complement activation compared with warfarin in antiphospholipid syndrome patients with venous thromboembolism. J Thromb Haemost 2016;14:2177-86.

24. Arachchillage DR, Efthymiou M, Mackie IJ, Lawrie AS, Machin SJ, Cohen H. Rivaroxaban and warfarin achieve effective anticoagulation, as assessed by inhibition of TG and in-vivo markers of coagulation activation, in patients with venous thromboembolism. Thromb Res 2015;135:388-93.

25. Arachchillage DR, Mackie IJ, Efthymiou M, Isenberg DA, Machin SJ, Cohen H. Interactions between rivaroxaban and antiphospholipid antibodies in thrombotic antiphospholipid syndrome. J Thromb Haemost 2015;13:1264-73.

26. Kunk P, Macik B, Rice M. Non-warfarin oral anticoagulants in anti-phospholipid syndrome. Blood 2015;126:1133.

27. Malec K, Góralczyk T, Undas A. The use of direct oral anticoagulants in 56 patients with antiphospholipid syndrome. Thromb Res 2017;152:93-7.

28. Son M, Wypasek E, Celinska-Lowenhoff M, Undas A. The use of rivaroxaban in patients with antiphospholipid syndrome: A series of 12 cases. Thromb Res 2015;135:1035-6.

29. Signorelli F, Nogueira F, Domingues V, Mariz HA, Levy RA. Thrombotic events in patients with antiphospholipid syndrome treated with rivaroxaban: a series of eight cases. Clin Rheumatol 2016;35:801-5.

30. Sciascia S, Breen K, Hunt BJ. Rivaroxaban use in patients with antiphospholipid syndrome and previous venous thromboembolism. Blood Coagul Fibrinolysis 2015;26:476-7.

31. Noel N, Dutasta F, Costedoat-Chalumeau N, Bienvenu B, Mariette X, Geffray L, et al. Safety and efficacy of oral direct inhibitors of thrombin and factor $\mathrm{Xa}$ in antiphospholipid syndrome. Autoimmun Rev 2015;14:680-5.

32. Betancur JF, Bonilla-Abadía F, Hormaza AA, Jaramillo FJ, Cañas CA, Tobón GJ. Direct oral anticoagulants in antiphospholipid syndrome: a real life case series. Lupus 2016;25:658-62.

33. Haladyj E, Olesinska M. Rivaroxaban - a safe therapeutic option in patients with antiphospholipid syndrome? Our experience in 23 cases. Reumatologia 2016;54:146-9.

34. Dufrost V, Risse J, Wahl D, Zuily S. Direct oral anticoagulants use in antiphospholipid syndrome: are these drugs an effective and safe alternative to warfarin? a systematic review of the literature: response to comment. Curr Rheumatol Rep 2017;19:52.

35. Burns PB, Rohrich RJ, Chung KC. The levels of evidence and their role in evidence-based medicine. Plast Reconstr Surg 2011;128:305-10.

36. Harter K, Levine M, Henderson SO. Anticoagulation drug therapy: a review. West J Emerg Med 2015;16:11-7.

37. Holbrook A, Schulman S, Witt DM, Vandvik PO, Fish $\mathrm{J}$, Kovacs MJ, et al. Evidence-based management of anticoagulant therapy: Antithrombotic Therapy and Prevention of Thrombosis, 9th ed: American College of Chest Physicians Evidence-Based Clinical Practice Guidelines. Chest 2012;141(2 Suppl):e152S-e84S.

38. Chighizola CB, Moia M, Meroni PL. New oral anticoagulants in thrombotic antiphospholipid syndrome. Lupus 2014;23:1279-82.

39. Kuruvilla M, Gurk-Turner C. A review of warfarin dosing and monitoring. Proc (Bayl Univ Med Cent) 2001;14:305-6.

40. Cohen H, Efthymiou M, Isenberg DA. Use of direct oral anticoagulants in antiphospholipid syndrome. J Thromb Haemost 2018;16:1028-39. 
41. Ruiz-Irastorza G, Hunt BJ, Khamashta MA. A systematic review of secondary thromboprophylaxis in patients with antiphospholipid antibodies. Arthritis Rheum. 2007;57:1487-95.

42. Efthymiou M, Lawrie AS, Mackie I, Arachchillage D, Lane PJ, Machin S, et al. Thrombin generation and factor $\mathrm{X}$ assays for the assessment of warfarin anticoagulation in thrombotic antiphospholipid syndrome. Thromb Res 2015;135:1191-7.

43. Kubitza D, Becka M, Voith B, Zuehlsdorf M, Wensing G. Safety, pharmacodynamics, and pharmacokinetics of single doses of BAY 59-7939, an oral, direct factor Xa inhibitor. Clin Pharmacol Ther 2005;78:412-21.

44. Gerotziafas GT, Elalamy I, Depasse F, Perzborn E, Samama MM. In vitro inhibition of thrombin generation, after tissue factor pathway activation, by the oral, direct factor Xa inhibitor rivaroxaban. $\mathrm{J}$ Thromb Haemost 2007;5:886-8.

45. Arachchillage DJ, Cohen $\mathrm{H}$. Use of new oral anticoagulants in antiphospholipid syndrome. Curr Rheumatol Rep 2013;15:331.

46. Ruiz-Irastorza G, Cuadrado MJ, Ruiz-Arruza I, Brey $\mathrm{R}$, Crowther M, Derksen R, et al. Evidence-based recommendations for the prevention and long-term management of thrombosis in antiphospholipid antibody-positive patients: report of a task force at the 13th International Congress on antiphospholipid antibodies. Lupus 2011;20:206-18.

47. Tripodi A, Chantarangkul V, Clerici M, Negri B, Galli M, Mannucci PM. Laboratory control of oral anticoagulant treatment by the INR system in patients with the antiphospholipid syndrome and lupus anticoagulant. Results of a collaborative study involving nine commercial thromboplastins. $\mathrm{Br} \mathrm{J}$ Haematol 2001;115:672-8.
48. Mueck W, Lensing AW, Agnelli G, Decousus H, Prandoni P, Misselwitz F. Rivaroxaban: population pharmacokinetic analyses in patients treated for acute deep-vein thrombosis and exposure simulations in patients with atrial fibrillation treated for stroke prevention. Clin Pharmacokinet 2011;50:675-86.

49. Cervera R, Serrano R, Pons-Estel GJ, Ceberio-Hualde L, Shoenfeld Y, de Ramón E, et al. Morbidity and mortality in the antiphospholipid syndrome during a 10-year period: a multicentre prospective study of 1000 patients. Ann Rheum Dis 2015;74:1011-8.

50. Patel MR, Mahaffey KW, Garg J, Pan G, Singer $\mathrm{DE}$, Hacke $\mathrm{W}$, et al. Rivaroxaban versus warfarin in nonvalvular atrial fibrillation. $\mathrm{N}$ Engl $\mathrm{J}$ Med 2011;365:883-91.

51. Cohen H, Hunt BJ, Efthymiou M, Mackie IJ, Khamashta M, Isenberg DA. Direct oral anticoagulants use in antiphospholipid syndrome: are these drugs an effective and safe alternative to warfarin? a systematic review of the literature: comment. Curr Rheumatol Rep 2017;19:50.

52. Win K, Rodgers GM. New oral anticoagulants may not be effective to prevent venous thromboembolism in patients with antiphospholipid syndrome. Am J Hematol 2014;89:1017.

53. Finazzi G, Marchioli R, Brancaccio V, Schinco P, Wisloff $F$, Musial J, et al. A randomized clinical trial of high-intensity warfarin vs. conventional antithrombotic therapy for the prevention of recurrent thrombosis in patients with the antiphospholipid syndrome (WAPS). J Thromb Haemost 2005;3:848-53.

54. Schaefer JK, McBane RD, Black DF, Williams LN, Moder KG, Wysokinski WE. Failure of dabigatran and rivaroxaban to prevent thromboembolism in antiphospholipid syndrome: a case series of three patients. Thromb Haemost 2014;112:947-50. 